\title{
A new optimum design method of bicycle parameters for a specified rider
}

The optimum design of bicycle parameters has been explored by many scholars and institutes since bicycles were first invented. Professor XinJun Liu and his group at Tsinghua University established a new way to design bicycle parameters according to the dimensions of the rider's body. They introduced a new perspective of the rider-bicycle system by considering the complete system as a mechanism. The group then established a new method for the optimum design of bicycle parameters from a completely theoretical basis, which may result in a new field of optimum design of bicycle parameters. Their work, entitled "Analysis and optimum design of rider-bicycle mechanisms: Design of bicycle parameters for a specified rider" was published in Science China Technology Sciences, 2011, Vol. 54(11).

Since bicycles were first invented, they have played a crucial role in people's lives. Thus, many scholars, engineers, and institutes have made efforts to optimize the design of bicycles. The tools that have been employed to optimize the bicycle have been based entirely on experimental and statistical results. For example, Henri and Bremner conducted experiments to measure the effect of parameters of the bicycle frame on the level of comfort experienced by riders. They developed a system for the commercial design of bicycles from their experimental results. Using a self-organizing-data excavating algorithm, Zhang from Beijing University of Technology analyzed experimental data for 210 riders and their bicycles, and proposed an empirical formula describing the relationship between the two datasets.

Professor Liu's group considered the issue of optimal bicycle design from a mechanical perspective. They first analyzed rider-bicycle mechanisms, and mechanized the system as a double-crank-and-rocker mechanism. Figure 1 is the sketch of a rider and a bicycle, which can be thought as a rider-bicycle mechanism such that the rider's buttocks are fixed to the seat and connected to the thighs through the hips, the thigh is linked to the shank by the knee, the shank is connected to the foot through the ankle, and the foot is attached to the crank of the bicycle. All the connections are considered as revolution pairs, and the thigh and crank are considered as bars. A double-crank-and-rocker mechanism is thus established. In such a mechanism, the thigh works as the driver and the vertical seat bar as the frame.

After mechanizing the rider-bicycle system, the group constructed a design space to calculate the length of the crank and the seat height (i.e., length of the vertical bar) with the constraint of geometry and the rider's physical dimensions. In the design space, they introduced the global transmission index (GTI) and global strain index (GSI) to describe the efficiency of force transition and the fatigue of the thigh. It is well known that the better the efficiency of the force transition is and the less the fatigue of the thigh is, the better the performance of the rider-bicycle system will be. The researchers revealed that the GTI increases with improving force transition and that GSI increases with less fatigue of the thigh.

Professor Liu's group contoured the GTI and GSI in the design space. That is, they obtained charts of GTI and GSI. Since the design space and the performance indices are related to the constraints of the dimensions of the rider's body, different riders with different ratios of the thigh and shank will have different charts of GTI and GSI. Thus, according to the requirements for the GTI and GSI, there will be an optimum region for the bicycle parameters (i.e., the crank length and seat height).

The group then established a design process based on their theoretical work as follows. First, the lengths of the thigh and shank of the rider are measured. Second, the contours of GTI and GSI are drawn using the lengths of the thigh and shank. Third, the optimum region is found according to the requirements of GTI and GSI. Finally, the design is taken from the optimum region and is verified.

After many years' research on the optimum design of a bicycle, scholars have established applicable empirical formulas. This research project explored a new way to optimize the design of bicycle parameters. The innovation of this work is the provision of a calculation method having a mechanical perspective. In this project, the structure of the human body (thigh and shank) and the crank length and seat height of the bicycle were mechanized. A double-crank-and-rocker mechanism was thus obtained as the bicycle-rider mechanism. From a mechanism perspective, a method to optimize the design of the crank and seat height of a bicycle according to different lengths of a person's thigh and shank was proposed. In this manner, a "customized bicycle" is realized. The result of the paper is useful for matching bicycles to athletes, bicycle manufacturing, and the design of cycling robots.

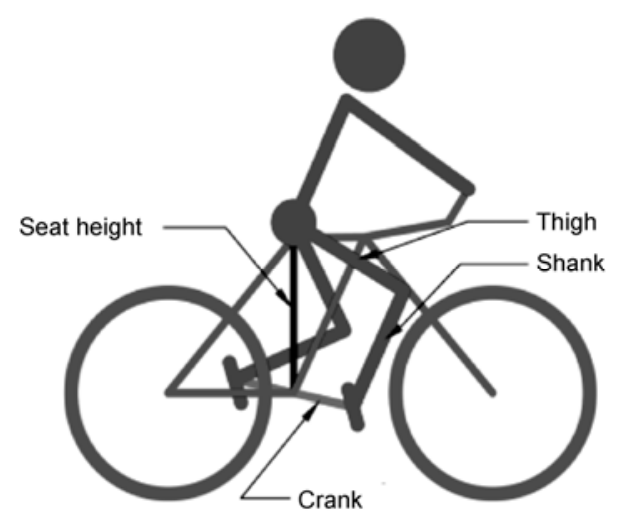

Figure 1 Sketch of a rider on a bicycle.

See the article: Liu X J, Wang J, Yu W Y, et al. Analysis and optimum design of rider-bicycle mechanisms: Design of bicycle parameters for a specified rider. Sci China Technol Sci, 2011, 54: 3027-3024

Open Access This article is distributed under the terms of the Creative Commons Attribution License which permits any use, distribution, and reproduction in any medium, provided the original author(s) and source are credited. 